\title{
Article
}

\section{The Michigan State University Twin Registry (MSUTR): 15 Years of Twin and Family Research}

\author{
S. Alexandra Burt and Kelly L. Klump \\ Department of Psychology, Michigan State University, East Lansing, MI, USA
}

\begin{abstract}
The primary aim of the Michigan State University Twin Registry (MSUTR) is to examine developmental differences in genetic, environmental, neural, epigenetic, and neurobiological influences on psychopathology and resilience, particularly during childhood and adolescence. The MSUTR has two broad components: a large-scale, population-based registry of child, adolescent, and adult twins and their families (current $N \sim 30,000)$ and a series of more focused and in-depth studies drawn from the registry (projected $N \sim 7200$ ). Participants in the populationbased registry complete a family health and demographic questionnaire via mail. Families can then be recruited for one or more of the intensive, in-person studies from the population-based registry, using any one of several recruitment strategies (e.g., population-based, based on their answers to the registry questionnaire). These latter studies target a variety of biological, genetic, and environmental phenotypes, including multi-informant measures of psychiatric and behavioral phenotypes, functional and structural neuroimaging, comprehensive measures of the twin family environment (e.g., census and neighborhood informant reports of twin neighborhood characteristics, videotaped interactions of child twin families), buccal swab and salivary DNA samples, and/or assays of adolescent and adult steroid hormone levels. This article provides an overview of the MSUTR and describes current and future research directions.
\end{abstract}

Keywords: twins; children-of-twins; environment; genetics; epigenetics; hormones; Michigan State University Twin Registry (MSUTR)

(Received 31 May 2019; accepted 12 July 2019; First Published online 30 August 2019)

The overall focus of the Michigan State University Twin Registry (MSUTR) is on understanding developmental changes in genetic, environmental, and neurobiological influences on internalizing and externalizing psychopathology and resilience. Several features of the MSUTR distinguish it from other twin registries. First, the registry takes a lifespan perspective by assessing twins during childhood, adolescence and adulthood, and via intensive longitudinal designs. This lifespan perspective ensures that genetic, environmental, and biological risk factors specific to particular developmental periods are identified and examined for their relevance for psychopathology and resilience. Second, there is a focus on understanding mechanisms of etiologic effects. While standard twin biometric model fitting analyses are used to examine relative contributions of genetic and environmental factors, these analyses are extended by the direct assessment of genes (e.g., via polygenic risk scores), measured environmental factors (e.g., parent-child interactions, neighborhood characteristics), neural development (e.g., functional and structural neuroimaging) and biological risk factors (e.g., steroid hormone concentrations). These extended assessments allow for the examination of genotype $\times$ environment $(\mathrm{G} \times \mathrm{E})$ interactions across multiple levels of analysis (i.e., neural, neurobiological, behavioral), as well as the mediation of genetic effects by neurobiological factors.

Author for correspondence: S. Alexandra Burt, Email: burts@msu.edu

Cite this article: Burt SA and Klump KL. (2019) The Michigan State University Twin Registry (MSUTR): 15 Years of Twin and Family Research. Twin Research and Human Genetics 22: 741-745, https://doi.org/10.1017/thg.2019.57
In the present article, we describe MSUTR recruitment methods, study assessments, and individual research projects.

\section{Recruitment Methods and Sample Representativeness}

Although the MSUTR initially began as a university-based twin registry assessing undergraduate men and women, we moved to recruiting twins via birth records beginning in 2004. The Michigan Department of Health and Human Services (MDHHS; formerly known as the Michigan Department of Community Health) identifies twin pairs residing in lower Michigan who meet our study age criteria (see criteria below) and whose addresses or parents' addresses (for twins who are minors) can be located either using driver's license information obtained from the state of Michigan or the proprietary search engine used by police (since the MDHHS, as the state agency in charge of vital records, has direct access to individual social security numbers (SSNs), full names, and birth dates). Twins are identified either directly from birth records or via the Michigan Twins Project (MTP), a largescale twin registry within the MSUTR that doubles as a recruitment resource for smaller, more intensive projects. Because birth records are confidential in Michigan, recruitment packets are mailed directly from the MDHHS to eligible twin-pairs to ensure their confidentiality. Twins indicating interest in participation via prestamped postcards or emails/calls to the MSUTR project office are then contacted by study staff to determine study eligibility and to schedule their assessments.

Four recruitment mailings are used for each study to ensure optimal twin participation. Overall, response rates across studies 
Table 1. Summary of existing MSUTR project assessments

\begin{tabular}{|c|c|c|c|c|c|c|c|c|c|}
\hline \multirow[b]{2}{*}{ Measure } & \multicolumn{8}{|c|}{ Study } & \multirow[b]{2}{*}{ HBMC- $f M R I$} \\
\hline & MTP & TBED-C & MTwins & Mindset & TSMBHP & TSMBHP-M & HBMC & $\mathrm{HBOC}$ & \\
\hline Zygosity determination & $\mathrm{x}$ & $\mathrm{x}$ & $x$ & $x$ & $x$ & $\mathrm{x}$ & $x$ & $x$ & $\mathrm{x}$ \\
\hline Internalizing symptoms (e.g., disordered eating) & $x$ & $x$ & $x$ & & $x$ & $x$ & $x$ & $x$ & $x$ \\
\hline Externalizing symptoms (e.g., antisocial behavior) & $x$ & $x$ & $x$ & & $x$ & $x$ & $x$ & $x$ & $x$ \\
\hline Parental personality and psychopathology & & $x$ & $x$ & & $x$ & & & & \\
\hline Family history of psychopathology & $x$ & & & & & & & & \\
\hline Twin temperament & & & $x$ & & & & & & \\
\hline Twin pubertal development & $x$ & $x$ & $x$ & & & & & & \\
\hline \multicolumn{10}{|l|}{ Twin steroid hormones ${ }^{a}$} \\
\hline Cortisol & & & $x$ & & & & & & \\
\hline \multicolumn{10}{|l|}{ Finger-length ratios } \\
\hline Neuroimaging & & & $x$ & & & & & & $x$ \\
\hline Twin birth complications & $x$ & & & & & & & & \\
\hline Use of fertility medications & $x$ & & & & & & & & \\
\hline Birth height and weight & $x$ & & & & & & & & \\
\hline Body mass index and/or $\%$ body fat & $x$ & $x$ & $x$ & & $x$ & $x$ & $x$ & $x$ & $x$ \\
\hline Parent-twin relationship & & $x$ & $x$ & & $x$ & $x$ & & & \\
\hline Neighborhood characteristics & $x$ & $x$ & $x$ & $x$ & & & & & \\
\hline Parent's marital relationship & & $\mathrm{x}$ & $x$ & & $x$ & & & & \\
\hline Peer deviance, teasing, prosociality & & $x$ & $x$ & & & & & & \\
\hline Sociocultural risk factors (e.g., media) & & & & & $x$ & $x$ & & & \\
\hline Stressful life events & & & & & & $x$ & $x$ & & \\
\hline Academic/verbal abilities & & $x$ & $x$ & $x$ & & & & & \\
\hline Twin DNA $^{\mathrm{b}}$ & & $x$ & $x$ & & $x$ & $x$ & $x$ & $x$ & $x$ \\
\hline Parent DNA ${ }^{b}$ & & $x$ & $x$ & & $x$ & $x$ & & & \\
\hline Epigenomewide methylation ${ }^{c}$ & & $x$ & $x$ & & & & & & \\
\hline Gene expression & & & $x$ & & $x$ & & $x$ & $x$ & \\
\hline
\end{tabular}

Note: MTP = Michigan Twins Project; TBED-C = Twin Study of Behavioral and Emotional Development - Child; MTwins = Michigan Twin Neurogenetics Study; TSMBHP = Twin Study of Mood, Behavior, and Hormones during Puberty; TSMBHP-M = Twin Study of Mood, Behavior, and Hormones during Puberty in Males; HBMC = Twin Study of Hormones and Behavior across the Menstrual Cycle; HBOC = Twin Study of Hormones and Behavior across Oral Contraceptive Use; HBMC = Twin Study of Hormones and Behavior across the Menstrual Cycle - fMRI study.

aSteroid hormones for each twin were assayed from salivary, finger-prick blood spot and/or serum samples.

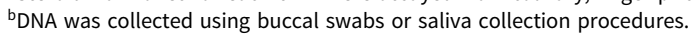

'Salivary and blood methylomes.

(56-85\%; see Figure 1) are on par with or better than those of other twin registries that use similar types of anonymous recruitment mailings, and have thus far yielded largely representative samples. Families recruited into the families of the naturally conceived twins in the large-scale MTP, for example, closely resemble families across the state of Michigan (Burt \& Klump, 2012). The proportion of MTP families that identify as White, non-Hispanic is $81.0 \%$, which is very similar to the $80.2 \%$ indicated in statewide census data. Mean family incomes are also quite comparable $(\$ 75,940$ in the MTP vs. $\$ 73,373$ in the census), as are the proportion of families with graduate or professional degrees $(10.3 \%$ in the MTP vs. $9.6 \%$ in the census).

These successes extend to our smaller, more intensive in-person studies as well. For example, the 529 population-based twin families and 502 at-risk twin families in the completed Twin Study of Behavioral and Emotional Development in Children (TBED-C) were generally representative of recruited but nonparticipating families. As compared to nonparticipating twins, participating twins reported similar levels of conduct problems, emotional symptoms or hyperactivity ( $d$ ranged from -.08 to 0.01 in the population-based sample and .01 to .09 in the at-risk sample; all $n s$ ). Participating families also did not differ from nonparticipating families in paternal felony convictions $(d=-.01-.13$ for the population-based and the at-risk samples, respectively), rate of singleparent homes $(d=.10$ and -.01 for the population-based and the at-risk samples, respectively), paternal years of education (both $d \leq .12$ ) or maternal and paternal alcohol problems ( $d$ ranged from .03 to .05 across the two samples). However, participating mothers in both samples reported slightly more years of education $(d=.17$ and .26 , both $p<.05)$ than nonparticipating mothers. Maternal felony convictions differed across participating and nonparticipating families in the population-based sample $(d=-.20 ; p<.05)$ but not in the at-risk sample $(d=.02)$. In short, our recruitment procedures thus appear to yield samples that are representative of both recruited families and the general population of the state of Michigan. 


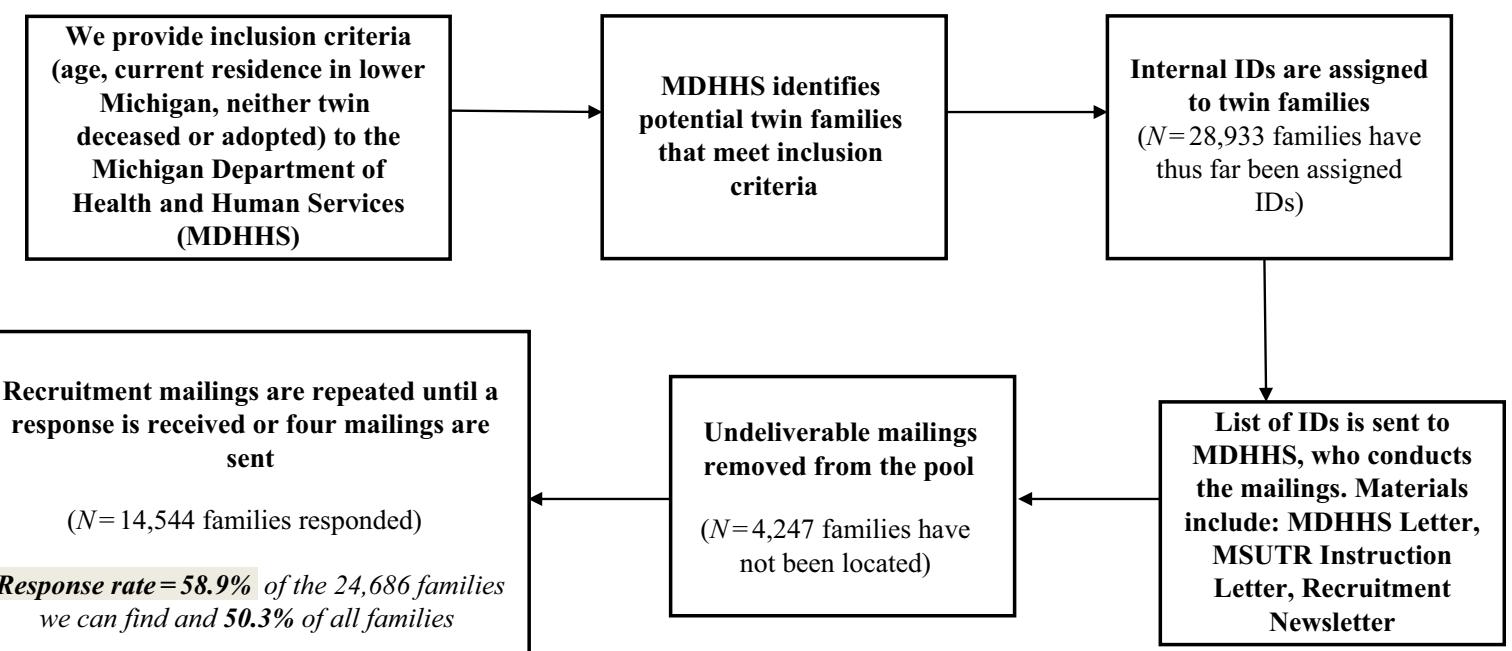

Response rate $=\mathbf{5 8 . 9} \%$ of the 24,686 families

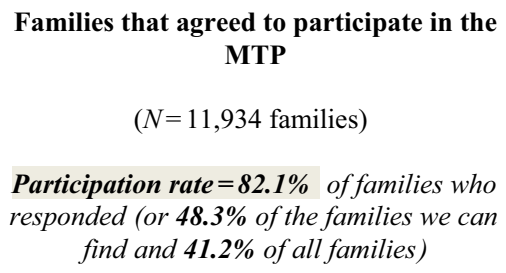

Fig. 1. Recruitment flow chart for the 3- to 17-year-old cohort in the Michigan Twins Project (MTP).

\section{Study Assessments}

Table 1 presents a summary of the assessments that comprise each of the individual MSUTR projects (projects described below). Given the relatively low base rates of internalizing and externalizing symptoms and the correspondingly large sample sizes needed for twin biometric modeling, there is an emphasis on continuous data in all of these projects (although structured diagnostic interviews are often included as well). There is also an emphasis on the use of developmentally appropriate measures. Finally, every effort is made to ensure that MSUTR projects use the same or similar measures of core phenotypes. This maximizes data collection efforts and allows for comparisons of etiologic effects across datasets and developmental periods.

Zygosity was established using physical similarity questionnaires (administered to the twins and/or their parents) that show accuracies of $95 \%$ or better (Iacono et al., 1999; Lykken et al., 1990; Peeters et al., 1998). For several projects, a research assistant (RA) also independently evaluates the twins on physical similarity indices. Zygosities are then compared between the participant and RA reports, and discrepancies are resolved through review of questionnaire data and twin photographs (when available) by one of the MSUTR principal investigators or by DNA markers.

\section{MSUTR Projects}

\section{Parent Twin Registry}

The MTP is an ongoing, population-based, mail-in registry of child and adolescent twins aged 3-17 years and their families, and adult twins aged 18 to 55 years ( 30,000 twins in $15,000+$ pairs; $~ 80 \%$ are in the younger cohort). We have also begun to reassess the younger twins roughly 5 years after their original participation (current $N \sim 800$ pairs). The brief questionnaire completed as part of participation in the MTP can be used to immediately generate new knowledge (Burt \& Klump, 2012; Racine et al., 2014), and indeed is included as part of the COllaborative project of Development of Anthropometrical, also described in this issue (Silventoinen et al., 2019). That said, the primary purpose of the MTP is to serve as a world-class 'participant bank' from which researchers can identify and recruit subsamples of twins and their families for genetically and environmentally informed research. The more focused, in-depth studies making use of this recruitment resource are described below.

\section{In-Depth Studies at Michigan State University (MSU)}

Twin study of emotional and behavioral development in children (TBED-C). The primary focus of the TBED-C was on understanding the role of $\mathrm{G} \times \mathrm{E}$ in the etiology of childhood-onset psychopathology. As noted above, the TBED-C includes both a population-based sample and an independent at-risk sample for which inclusion criteria also specified that participating twin families lived in neighborhoods with neighborhood poverty levels at or above the census mean at study onset (10.5\%). Twins in the TBED-C ranged in age from 6 to 10 years (mean $=7.7, S D=1.51$; although 27 pairs had turned 11 by the time the family participated) and were $48.7 \%$ female. The sample includes 224 monozygotic (MZ) male pairs, 211 dizygotic (DZ) male pairs, $202 \mathrm{MZ}$ female pairs, 206 DZ female pairs and 187 DZ opposite-sex pairs. 
In service to our core aim, we measured multiple aspects of the twins' environment, with a particular focus on parent-child relationships, structural and social characteristics of the twins' neighborhoods and the parents' marital relationship. The TBED-C has already produced $\sim 40$ peer-reviewed publications (e.g., Burt et al., 2015; Burt et al., 2016; Burt et al., 2019; Burt et al., 2018), with more under way.

Michigan twin neurogenetics study (MTwins). MTwins is an ongoing study that follows the at-risk twins in the TBED-C, conducting two in-person twin family assessments during adolescence (18-24 months apart), beginning 4-6 years after their prior TBED-C assessment. Our target sample sizes are 500 families at time 2 (early adolescence, aged 10-17 years) and 400 families at time 3 (mid-adolescence, aged 11-18 years). Records and dried blood spots from the twins' pre/neonatal stage are also available. Assessments at both time points include task and resting functional and structural neuroimaging (fMRI, DTI and sMRI), thereby allowing us to identify the neural regulatory control architecture (RCA) associated with neighborhood disadvantage, to evaluate whether and how the etiology of neural RCA varies with disadvantage.

Mindset study. The Mindset Study constitutes the first randomized intervention embedded in a behavioral genetic design (to our knowledge). We are recruiting 800 adolescent twin pairs for a 1.5 -h online assessment conducted via Qualtrics. Twins are randomly assigned to either the experimental (growth mindset) or active control conditions, allowing us to directly evaluate whether and how a growth mindset induction alters the etiology of achievement-related phenotypes. It is hoped that this project will allow us to simultaneously consider and empirically integrate the (seemingly opposed) concepts of high heritability and malleability and provide empirical research relevant to key philosophical discussions about the meaning of heritability and the goals of human behavioral genetics.

Adolescent twin study of behavioral adjustment and development (Adolescent-TSBAD). The primary focus of the Adolescent-TSBAD is to identify gonadal hormone regulation of individual differences and sex differences in pubertal risk. These aims are achieved via in-depth assessments of internalizing and externalizing symptoms, pubertal development, genes (via genomewide association analyses) and gonadal and adrenal hormone concentrations (e.g., estrogen, progesterone, testosterone, dehydroepiandrosterone (DHEA)), in addition to several psychosocial risk factors (e.g., teasing, parent-child relationships). Two of the largest Adolescent-TSBAD studies include the Twin Study of Mood, Behavior, and Hormones during Puberty (TSMBHP), which assessed $996(M Z=480$ [48\%]; $D Z=516$ [52\%]) female twins aged 8-16 years $(M=11.75, S D=2.03)$, and the ongoing TSMBHP-M, which will assess 1000 male twins aged 7-16 years. In combination, these projects will allow for the most in-depth, developmental examinations of $\mathrm{G} \times \mathrm{E}$ interactions for psychopathology both within and across sex. To date, Adolescent-TSBAD studies have generated over 20 publications, with several more in preparation.

Adult twin study of behavioral adjustment and development (Adult-TSBAD). Nearly all of the Adult-TSBAD studies use intensive, daily data collections of disordered eating, mood, substance use and salivary samples of ovarian hormones as quasi-experimental designs for identifying dynamic changes in genetic, environmental and hormonal risk for psychopathology across extended time periods (i.e., 45-49 consecutive days). The largest Adult-TSBAD study to date is the Twin Study of Hormones and Behavior across the Menstrual Cycle that conducted daily assessments of 650 female twins (57.5\% MZ, 42.5\% DZ; aged 16-25 years), although another ongoing study, the Twin Study of Hormones and Behavior across Oral Contraceptive Use, is completing daily assessments of 1000 female twins who are concordant or discordant for oral contraceptive use. Finally, an ongoing, smaller study (the Twin Study of Hormones and Behavior - fMRI study; target $N=24$ ) is substantially extending these findings by conducting repeated fMRI scans of female twins to identify the intermediary, neural systems that may mediate ovarian hormone and genetic effects on psychopathology in women. In total, these studies have generated over 30 publications, with more underway.

\section{External Studies}

In addition to the above, several researchers external to MSU are also making use of the MSUTR as a recruitment resource for their own indepth studies. As one example, the Twin Study of Attention (Principal Investigator: Joel Nigg) aims to identify epigenetic biomarkers for Attention Deficit Hyperactivity Disorder (ADHD), thus far enrolling $175 \mathrm{MZ}$ pairs. The Epigenomics of Hypertension in Monozygotic Twins and Effect of Salt Intake aims to examine epigenetic effects on hypertension (Principal Investigator: Srivdya Kidambi, MD) and has enrolled 108 adult MZ pairs in the study. Results from these projects are forthcoming.

\section{Future Directions}

We are hopeful that the developmentally informed approach of the MSUTR will continue to yield data that clarifies the role of genetic, environmental, neural, epigenetic and neurobiological factors in the development of psychopathology across the lifespan. We welcome collaborations with other twin researchers and registries on both new and existing MSUTR projects. Please see https:// msutwinstudies.com/msutr-data for more details on collaborations. Through such collaborations, we hope to further increase understanding of the etiologies of psychopathology and resilience across the lifespan.

Acknowledgments. This work was supported by the following: R01MH081813, R01-MH0820, R01-MH 092377, R21-MH070542, R03 MH63851, UG3/UH3-MH114249, R01-MH111715 and R01-MH118848 from the National Institute of Mental Health (NIMH), R01-HD066040 and R01HD093334 from the Eunice Kennedy Shriver National Institute for Child Health and Human Development (NICHD), \#11-SPG-2408 from Michigan State University, The Avielle Foundation's Conway Family Award for Excellence in Neuroscience, and a Global Foundation for Eating Disorders Grant. The content is solely the responsibility of the authors and does not necessarily represent the official views of the NIMH, the NICHD or the National Institutes of Health.

\section{References}

Burt, S. A., Donnellan, M. B., Slawinski, B. L., \& Klump, K. L. (2015). The phenomenology of non-aggressive antisocial behavior during childhood. Journal of Abnormal Child Psychology, 44, 1-11.

Burt, S. A., \& Klump, K. L. (2012). How does the inclusion of twins conceived via fertility treatments influence the results of twin studies? Twin Research and Human Genetics, 15, 746-752. 
Burt, S. A., Klump, K. L., Gorman-Smith, D., \& Neiderhiser, J. M. (2016). Neighborhood disadvantage alters the origins of children's non-aggressive conduct problems. Clinical Psychological Science, 4, 511-526.

Burt, S. A., Pearson, A. L., Rzotkiewicz, A., Klump, K. L., \& Neiderhiser, J. M. (2019). It really does take a village: The role of neighbors in the etiology of non-aggressive rule-breaking. Development \& Psychopathology, 31, 713-725.

Burt, S. A., Slawinski, B. L., \& Klump, K. L. (2018). Are there sex differences in the etiology of youth antisocial behavior? Journal of Abnormal Psychology, 127, 66-78.

Iacono, W. G., Carlson, S. R., Taylor, J., Elkins, I. J., \& McGue, M. (1999). Behavioral disinhibition and the development of substance use disorders: Findings from the Minnesota Twin Family Study. Development and Psychopathology, 11, 869-900.
Lykken, D. T., Bouchard, T. J., McGue, M., \& Tellegen, A. (1990). The Minnesota Twin Family Registry: Some initial findings. Acta Gemellogicae et Medicae, 39, 35-70.

Peeters, H., Van Gestel, S., Vlietinck, R., Derom, C., \& Derom, R. (1998). Validation of a telephone zygosity questionnaire in twins of known zygosity. Behavior Genetics, 28, 159-161.

Racine, S. E., Culbert, K. M., Burt, S. A., \& Klump, K. L. (2014). Advanced paternal age at birth: Phenotypic and etiologic associations with eating pathology in offspring. Psychological Medicine, 44, 1029-1041.

Silventoinen, K., Jelenkovic, A., Yokoyama, Y., Sund, R., Sugawara, M., Tanaka, M., ... Kaprio, J. (2019). The CODATwins project: The current status and recent findings of COllaborative project of Development of Anthropometrical measures in twins. Twin Research and Human Genetics. Advance online publication. 